was in the left side of the scrotum. His age was 64. On the 24 th he had violent vomiting, had no stool, but his pulse was not affected. He had not much pain in the tumor or abdomen.

On the 25th the sickness was incessant, but still his strength kept up; his julse was but little quickened; and the abdomen was not tense. On the evening of this day he had some hiccough.

On the 26th the belly became tense; the pulse very frequent; he seemei excessively low; was still sick, and had frequent hiccough.

On the 27 th he was more low, but was only sick when he was moved; his pulse 120, and small; belly tense; roice low and feeble; mouth dry.

Operation employed, as all the common means had been employed without success. Intestine down, which felt cold, but little discoloured.

The stricture as firm as cartilage.

Pulse rose after the operation, and the body became warmer than before. No stools, however, followed, nor did the tension of the alsdomen decrease.

On the 28th, his pulse 140 , and very small. At noon he died.

On inspecting the body, the intestines appeared generally inflamed, as much so as the portion of colon which had protruded.

This man died from the operation being too long delayed.

In an old man, where there is appearance of sinking, the operation never succeeds, although it does sometimes in the younger.

\section{CASE OF ACUTE LARYNGITIS.}

TO 'THE EDITORS OF THE FROVINCIAL MEDICAL AND SURGICAL JOURNAL.

Gentremen,-I beg to forward the history of the following case of acute laryngitis, and if you consider it deserving a place in your pages, you will thereby oblige your obedient servant,

\section{Jordan Roche Lynch,}

M.D. M.R.C.S.L.

Medical Officer of the West London Union.

John Whaler, aged 53, a labourer, lodging at 56 , West-street, a very powerful man, of temperate habits, latterly had suffered severe privations from want of employment. He was attacked, aftcr exposure to wet, about noon on the 3rd inst., with pain, tenderness, and sense of constriction of the larynx; he went home, and applied in the evening for a relieving officer's order for medical assistance. I attended on its receipt, and found him labouring under all the symptoms of acute laryngitis.

The face was flushed; the skin hot; the pulse full and hard; countenance anxious in the extreme; the eyes staring; the nostrils raised; the voice reduced to a whisper, and articulation very difficult. The long deep shrill stridulous inspiration was so marked as to be heard outside the room, and so characteristic, that if once heard, it can never be forgotten. On examination of the fauces, there was no appearance to indicate the extent of mischief going on, but there was great enderness along the larynx. He was bled to ${ }_{3} x v i$.
The blood dark, thick, and flowed pleno rivo. As he had been reduced by insufficient nutriment, I was cautious as to the quantity abstracted. He was ordered hydrarg. chlorid. 3ss.; pul. opii. antim. tartar. $a a$. grs. ii., to be divided into six powders, one every half hour. I saw him again in an hour. No relief had been experienced. 'The act of swallowing caused convulsive fits of coughing, with frequent attempts to bring up a thick ropy mucus; difficulty of breathing increased; very restless, and every four or five minutes changing his position, in the vain hope of procuring ease. If he tried to lie down, he started up immediately, gasping, and in the greatest agitation, respiration every moment becoming a more convulsive struggle. I again bled him to 16 ounces, from which he expressed some slight relief; a copious perspiration burst out on head and trunk; the pulse became softer, and I began to hope for some mitigation of the intensity of the attack. The dysphagia, however, was so great, that he could not swallow a drop of water.

After turning out with some difficulty several of his friends who had flocked around his bed, and opening the window to cool and purify the atmosphere, I ordered twelve leeches to be applied, with mercurial inunction in both axillæ; I then called on my friend Mr. Waltham, one of the house-surgeons of St. Bartholomew's Hospital, in whose judgment, from his indefatigable pursuit of science, I have a high opinion, and requested him to accompany me, as I resolved upon performing bronchotomy.

On Mr. Waltham seeing him, the pulse was softand full, with profuse diaphoresis; the breathing and other symptoms as urgent as before. $\mathrm{Mr}$. Waltham suggested the propriety of removing him at once to the hospital, in order to give the benefit of a warm-bath, and of better attendance after the operation. I consented to this arrangement.

On admission, he was seen by the resident medical officer, Mr. Harlock, who prescribed the warm-bath, and twenty leeches to be applied to the throat, with hydr. chlorid. Эi.; pot. antim. tart. grs. iii., to be divided into six doses, one to be taken every half hour.

At ten o'clock the next morning there was not the slightest amendment; on the contrary, the patient was much more depressed; the difficulty of breathing continuing in the same state as when he was admitted. At 11 o'clock Dr. Roupell saw him, and promptly decided on the necessity of an artificial opening to his lungs being made; but before the arrival of the surgeon, which was not more than an hour and a half after the decision, a great change for the worse came on, so that while his trachea was being opened, he was expiring; artificial respiration was kept for some minutes after the operation, but without avail.

I attended the post-mortem examination. Dr. Roupell gave a short and very accurate history of the case. Mr. Paget carefully removed the larynx and pharynx. The following appearances presented themselves. The epiglottis was erect, thickened, and in such a state as to be incapable of protecting the glottis from the contact of matter passing into the pharynx. The mucous membrane was of an uniform dark-red colour; the folds forming the rima glottidis were very much thickened, and effusion had taken place in the submucous tissue, 
and beneath the fine lining membrane, which is reflected over the thyro-arytenoid ligament, and which sinks in between the superior and inferior ligaments, forming the ventricle of the larynx ; in this situation, the matter effused appeared to be sero-purulent. On bringing the sides of the chink of the glottis in contact, the natural opening was completely obliterated by the thickening, which prevented respiration, and was the obvious cause of death. There was no false membrane; it was clearly that form of disease to which the term laryngitis is properly restricted. I should have stated that the lungs were congested in the lower portion of the lobes, and the superior portion was filled with air, but not emphysematous.

\section{REMARKS.}

The prognosis of acute laryngitis is always unfavourable. Dr. Cheyne considered it the most fatal of all inflammations. The only hope, in many cases, is in giving relief by the operation. Upon this point, however, there exists much difference of opinion among medical authorities. Dr. Baillie was of opinion that blood-letting was of no use, and that tracheotomy should not be resorted to within less than thirty hours. In this case, death relieved the patient of his sufferings in less than that time. Dr. Armstrong mentions two cases that terminated fatally in seven and eight hours.

Mr. Lawrence says that bronchotomy should be performed as soon as the symptoms enable us to decide upon the nature of the disease, and wisely makes the condition of the patient more the criterion than the period of the attack.

Louis observes, that as long as bronchotomy is considered an extreme measure, it will be performed too late.

Drs. Mackintosh and Elliotson are in favour of the operation, but recommend previous trial of venesection and mercury.

This is very good advice in the first stage, before effusion has taken place; but in such a fell disease, when the sentinel of the portal of life is being overpowered, delay is death. The danger from the operation cannot be compared with the danger from the obstruction to the breathing, which it is calculated to remove. It is of no use to have recourse to it when the face becomes livid, and the faculties obtuse, from the circulation of black blood on the brain. The operation of bronchotomy is less liable to add to the irritation than that recommended between the crycoid and thyroid cartilages. It is also of a more simple nature. It is further removed from the seat of inflammation, and the mucous membrane is here endowed with less sensibility.

Some time ago, in a case in some respect similar to that just detailed, I cut down upon the trachea, in the manner described by Mr. Lawrence in his lectures, and introduced a very short canula, nearly approximating to the shape of that of M. Bretonneau, a description of which is given in plate 25, fig. 4, of M. Bourgery, and persevered with calomel and opium, and the application of leeches to the fauces, although the canula caused a good deal of irritation. The opening closed on about the 18th day, and the patient recovered.

In every case, as soon as the nature of the disease has fully manifested itself, bronchotomy should be performed; or an excision of one of the rings of the trachea, as described by Sir P. Crampton, should be made.

The tenderness of the larynx, and the peculiar inspiration, quite different from the hoarseness which often accompanies severe bronchitis, and which is caused by mucus or catarrhal inflammation, are the two pathognomonic signs quite sufficient to justify the use of the knife, and afford to your patient the only certain means of saving his life.

Medical men are, by their exposure to vicissitudes of temperature, peculiarly liable to this complaint; several of the greatest eminence have died of it. They should be always mindful of the saying of an old and great authority-"Celerrime intereunt cynanche laborantes, nonnunquam et antequam medicum accersiverint."

$$
\text { 9, King-street, Snow Hill, Sept. 14, } 1841 .
$$

\section{AMAUROSIS CURED BY STRYCHNIA.}

TO THE EDITORS OF THE PROVINCIAL MEDICAL AND SURGICAL JOURNAL.

Genthemen,-The following case is a short history of amaurosis occurring after sudden grief, but where other causes appear to have concurred in the production of the malady. Considerable relief was obtained by the repeated application of leeches, and the cure completed during the administration of strychnia.

If it be worthy of a place in your pages, I shall feel obliged by its insertion.

Yours respectfully,

J. Nottingham.

Liverpool, Nov. 15, 1841.

William M'Cole, aged 26, robust, eyes and complexion dark, a shoemaker, working constantly upon the polished or patent leather, "always enjoyed good health until the beginning of August last."

In this way he speaks of his previous health, but from more careful inquiry it appears that he has been subject to frequent epistaxis from the left nostril during the last six years, bleeding "on the average not less than once a week," but that a few weeks ago this periodic hæmorrhage ceased.

He remarks that he has been in the habit of attending to the state of his bowels, and of taking occasional purgatives; and although he has worked with the polished leather during the last two years, his eyes were never made uneasy, or pained, by its dazzling surface.

In July last, in consequence of the sudden death of his father, great anxiety about a family, numerous and unprovided for, was all at once brought upon him.

He describes his grief for the loss of his father, and his despair for the future prospects of his family, as having been exceedingly oppressive, and he himself regards these as the main cause of his malady; and it would seem that, taken together with the suppressed hæmorrhage above mentioned, they may account for the phenomena about to be detailed.

August 20, 1841. He applied to me with the following syptoms: Anxious expression of coun- 